\title{
Penile prosthesis biofilm formation and emerging therapies against them
}

\author{
Amin S. Herati ${ }^{1}$, Eric M. Lo ${ }^{2}$ \\ ${ }^{1}$ The James Buchanan Brady Urological Institute and Department of Urology, Johns Hopkins University School of Medicine, Baltimore, MD, USA; \\ ${ }^{2}$ Scott Department of Urology, Baylor College of Medicine, Houston, TX, USA \\ Contributions: (I) Conception and design: AS Herati; (II) Administrative support: AS Herati; (III) Provision of study material or patients: AS Herati; \\ (IV) Collection and assembly of data: AS Herati; (V) Data analysis and interpretation: AS Herati; (VI) Manuscript writing: All authors; (VII) Final \\ approval of manuscript: All authors. \\ Correspondence to: Amin S. Herati, MD. Brady Urological Institute, Department of Urology, Johns Hopkins School of Medicine, 4940 Eastern \\ Avenue, 301 Building, Suite 3112, Baltimore, MD 21224, USA. Email: aherati1@jhmi.edu.
}

\begin{abstract}
Infections are among the most feared and devastating complications of penile prosthesis infections, often requiring surgical exploration and explantation are prosthesis infections. While the rate of infections have decreased due to antibiotic prophylaxis, antiseptic device preparation, increased sterility in implantation techniques and device modifications, infections still occur at a rate of $1-3 \%$. This article reviews the formation of biofilms on penile prostheses and novel, experimental methods to prevent and eradicate them.
\end{abstract}

Keywords: Adult; biofilm; infection; penile prosthesis; treatment

Submitted Feb 07, 2018. Accepted for publication Sep 11, 2018.

doi: 10.21037/tau.2018.09.05

View this article at: http://dx.doi.org/10.21037/tau.2018.09.05

\section{Introduction}

Erectile dysfunction (ED) affects up to 152 million men worldwide (1). Current treatment options include oral phosphodiesterase-5 (PDE-5) inhibitors, intraurethral pellets, intracavernosal injections (ICIs), and penile prosthesis placement. Of these treatments, PDE-5 inhibitors remain the first-line option due to their efficacy and safety (2). Intraurethral pellets and ICIs are considered second-line therapies, while prostheses are considered definitive interventions as patients undergoing inflatable penile prosthesis (IPP) placement are either unable to tolerate, non-responsive, or refuse the aforementioned treatment options (3).

Although more expensive than their non-mechanical counterparts, IPPs offer a durable, concealable, and reliable mechanism for obtaining the rigidity necessary for intercourse with 15-year revision-free survival and satisfaction rates as high as $59.7 \%$ and $98 \%$, respectively (4). Despite the advantages, analysis of Medicare claims data from 2001 to 2010 shows decreasing utilization of IPPs (1). Lee et al. (1) identified a $50 \%$ decrease in utilization of IPPs across all demographic factors, such as age, ethnicity, and geography, despite a $165 \%$ increase in the incidence of ED. This decrease has occurred despite increasing public awareness of ED. However, direct-to-consumer marketing may have led to the higher relative use of medical therapy over prostheses placement in treating less severe ED (5). Interestingly, when IPPs were used, the surgeries were more likely to be performed on sicker patients with significantly more comorbidities (1). This finding may reflect the sicker patients' inabilities to respond to medical therapies. The increasing burden of comorbidities carries with it higher rates of intraoperative and postoperative complications $(6,7)$.

One of the most feared complications of IPP placement is infection, often requiring device removal. Antibiotic prophylaxis, antiseptic device preparation, improved implantation techniques and device modifications have reduced the rate to $1-3 \%$ (8). Contamination of the implant prior to or during the operation leads to planktonic 


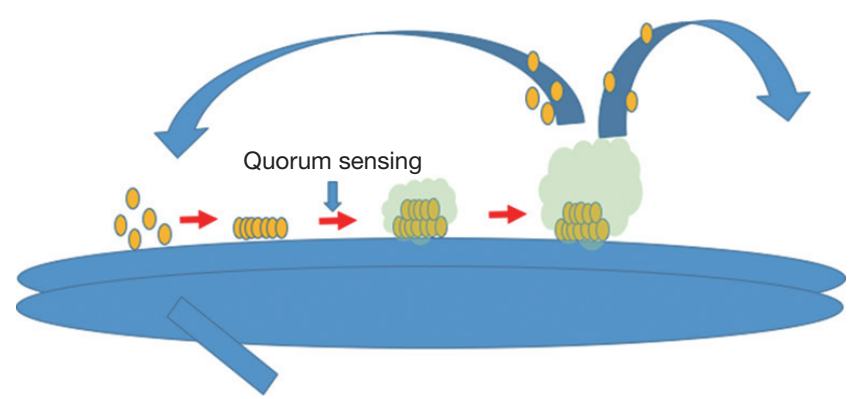

Figure 1 Four-step development of biofilm: (I) attachment of planktonic cells; (II) aggregation/accumulation of planktonic cells to form monolayer and microcolonies; (III) maturation; and (IV) detachment and dispersion.

organism proliferation and potential biofilm formation (9). Biofilms are colonies of bacteria or fungi that are capable of forming on variable surfaces such as in vivo medical devices, pipes and teeth. They can form on abiotic surfaces within 16 hours of device placement and insulate the causal organism from host immunologic defenses and antimicrobials $(10,11)$. Additionally, biofilms enhance microbial survival by reducing bacterial growth rate and promoting antimicrobial resistance, damage surrounding tissues, and trigger inflammation (12). In this article, we review factors contributing to biofilm formation on IPPs and novel methods to prevent and eradicate them.

\section{Microorganisms that form biofilms on penile prostheses}

Advancements in implant technology and antiseptic device preparation have decreased infection rates to approximately $1-3 \%$ in recent years (13). The two producers of 3-piece IPPs in the US have developed infection-retardant coatings on their implants to reduce infection rates. Boston Scientific (Marlborough, MA) uses a coating of rifampin and minocycline called InhibiZone ${ }^{\circledR}$. Coloplast Corporation (Humlebaek, Denmark) coats its devices with polyvinylpyrrolidone, a hydrophilic substance that absorbs the antibiotics the IPP is bathed in immediately prior to implantation (10). In a large observational study comparing the infection rates of 1,944 non-coated to 2,261 InhibiZone ${ }^{\circledR}$-coated IPPs, Carson reported a $50 \%$ reduction in infections at 180 days in the coated group (14).

Alterations have also been made to the operating room environment to reduce aerosolized bacteria, including laminar airflow systems and positive pressure devices developed by Brantley Scott (9). To further improve outcomes and the risk of infection, many surgeons have also adopted the "no touch" technique during procedures, as popularized by Eid (15). In this method, the implant, the instruments, and the surgeon's hands never make contact with the patient's skin during the operation. When paired with infection retardant coated IPPs, this "no touch" technique further reduces the rate of infection to $0.46 \%$ $(15,16)$.

Despite these advances in technology and technique, infections continue to occur. In a recent multicenter investigation of organisms cultured at the time of IPP salvage or explant, 204 organisms were identified (17). The three most prevalent organisms cultured were Escherichia coli $(18.3 \%)$, followed by coagulase-negative Staphylococcus species (15\%), and Candida species (11.1\%).

\section{Biofilm formation}

Biofilm formation can be divided into three different phases (Figure 1): (I) attachment; (II) maturation; and (III) dispersion [reviewed by Bjarnsholt et al. (18)]. In the first phase, planktonic cells attach to a surface and generate a microcolony through clonal growth (19). Once the microcolony has matured, the microbes secrete an insoluble three-dimensional matrix of extrapolymeric substances (EPS, e.g., polysaccharides, proteins, glycolipids, and extracellular DNA) that encase the microbes. Water channels are interspersed throughout the matrix, which permit the distribution of nutrients and oxygen (18). The clonal microbial growth and matrix expansion are highly regulated processes with variable growth patterns, suggesting possible genetic on-off switches. Transcriptome data from $P$. aeruginosa biofilms, however, suggest biofilm development depends more on the microenvironment's nutrient stores, such as glucose, iron and oxygen (20). The final phase of biofilm formation is dispersion, where microbes are released from the biofilm. This can occur either through mobilization of individual bacteria via genetically programmed secretion of enzymes [such as dispersin B (21)] or shearing of biofilm segments allowing sub-colonies to spread.

The trigger of microbial colonies to create biofilms is largely dependent on the environment (e.g., subinhibitory concentrations of antibiotics and the presence of pigments and iron siderophores) (22). Intricate communication systems between adjacent bacteria allow purposeful alterations in colony structure and function, including quorum sensing, chemotactic signaling and plasmid 
exchange [reviewed by Ben Jacob et al. (23)]. In quorum sensing, cell-to-cell communication synchronizes clonal behavior based on microbial density and nutrient supply. Cell-to-cell communication can also occur through the production of signaling molecules called autoinducers, which manipulate the gene expression of other intra-and interspecies bacteria (24).

Biofilms play an important role in the spread of antimicrobial resistance. The principle mechanism for this is thought to be horizontal transfer of resistance and virulence genes (25). While the mechanisms by which subinhibitory antibiotic concentrations promote the formation of biofilms remain unclear, one potential mechanism in Pseudomonas aeruginosa is through induction of the aminoglycoside response regulator (arr) gene (26). Increased arr expression resulted in activation of cyclic di-GMP signaling cascade and biofilm formation, while arr mutants were unable to form biofilms in response to subinhibitory concentrations of tobramycin. Disruption of this communication signaling may provide an avenue for biofilm disruption in the future.

\section{Biofilm therapy}

It is well accepted that intravenous antibiotics and/or prolonged courses of oral antibiotics without device removal are ineffective primary therapies for clinically infected IPPs. According to the 2015 recommendations of the International Consultation on Sexual Medicine, attempts should be made to remove all device components in a stable patient with an infected prosthesis and either immediately reimplant another IPP or replace it at a later date. During the explant, steps are taken to remove and disrupt any residual biofilm through parental antibiotics, vigorous antibiotic irrigation of the tissue surrounding the device, and attention to sterile technique. This is not without limitation, however. One-stage and twostage salvage procedures are associated with an increased risk of post-operative infection, penile fibrosis and penile shortening. Compared to the $1-3 \%$ infection rate of initial penile implants, the risk of reinfection increases to $10 \%$ for all salvage surgeries and up to $18 \%$ among diabetic men (27). Novel treatment strategies are therefore needed to address these biofilms. Various strategies will be discussed in the subsequent chapters based on their mechanism of disruption and their location of action.

\section{Prevention of microbial attachment}

A number of different strategies are available that make biologic surfaces inhospitable to microbes. Indeed, the primary strategy to prevent IPP infection is by inhibiting microbial attachment through the use of an anti-infective biomaterial coating. For example, coated implants from Boston Scientific and Coloplast Corporation utilize a hydrophobic layer to create a physical barrier to microbial attachment (28). While many other anti-infective strategies presented in the subsequent paragraphs carry the potential to decrease biofilm formation, their use is speculative and may never be utilized in penile prosthesis material as clinical efficacy testing is lacking.

Microbial surface binding depends on multiple variables that influence adhesion and colonization efficiency, such as the surface shape and chemical properties, environmental conditions, and pathogen specific factors [as reviewed by Campoccia et al. (29)]. Non-charged, hydrophilic and hydrophobic surfaces repel microbes floating in proteinrich solutions, such as blood or sera (29). The surfaces also prevent protein attachment, which further impairs bacterial adhesion. Altering the electrical charge of a surface prevents certain proteins in protein-rich solutions from attaching as binding to a hydrophobic or hydrophilic surface requires proteins to undergo a conformational change and disrupts the hydrogen bonds that normally allow hydrophobic segments of proteins to bind to hydrophobic surfaces (30). Thus a change in surface hydrophobicity increases the dependence of electrostatic charge for protein binding. An example of this strategy includes the use of a heparin coating. The application of a heparin coating increases the hydrophobicity of a solid surface and has been used to reduce bacterial adhesion to foley catheter surfaces and intraocular lenses (31-33). While heparin coatings have not been used on penile prostheses, heparin-coated ureteral stents have been tested against common uropathogens including Escherichia coli, Klebsiella pneumoniae, Enterococcus faecalis, Staphylococcus aureus, and Pseudomonas aeruginosa. Following a seven day in vitro exposure in a study by Lange et al. (34), heparin-coated ureteral stents did not decrease bacterial adherence and exhibited mature biofilm formation. Nevertheless, heparin-coatings may protect penile prostheses from biofilm formation through the interference of S. epidermidis adhesins binding to fibronectin (35).

Proteins can also be prevented from surface binding by creating morphologic barriers to their attachment. In vitro, smoothness down to the nanometer level, such as those seen on glass surfaces, is associated with reduced Gram-positive and Gram-negative bacterial adhesion (36). In contrast to nanometer scale smoothness, orthopedic implants have 
gained from the addition of micro-porous calcium phosphate coating laden with anti-microbial peptides (AMP) (37). These AMPs are short (12-40 amino acid in length), cationic and hydrophobic proteins with broad bactericidal activity (38). A limitation of micro-porous AMP loaded surface is its short-term duration of activity, which may limit its use in penile prostheses. A longer-term alternative to this approach maybe the use of quantum-sized materials called carbon nanotubes (CNT), which exhibit cytotoxicity to bacteria through the creation of oxidative stress and perturbation of the bacterial cell membrane (39). Other antimicrobials fixed to solid surfaces have been tested, including triclosan, chlorhexidine, nitric oxide releasing polymers, coatings release reactive oxygen species, and more recently thermal stress inducing superparamagnetic iron oxide nanoparticles (29). Again, this technology has not yet been utilized with penile prostheses.

Biologic approaches may be utilized to mitigate the clinical burden of biofilm formation on penile prostheses. Similar to other commensal bacteria in our bodies that safeguard against the proliferation of pathologic bacteria, certain bacteria may play a protective role in the adhesion and colonization of other pathogenic bacteria. In a recent analysis of twelve penile prostheses removed due to mechanical failure, Etcheverry-Giadrosich et al. (40) found five prostheses colonized with S. epidermidis without clinical infection. Biosurfactant produced by these probacteria may inhibit attachment of other virulent strains of bacteria $(41,42)$. It's unclear of the use of probacteria will reach clinical practice as $S$. epidermidis remains a pathogen commonly implicated in the development of IPP infections.

\section{Inhibition of microcolony formation through extracellular polymeric substance disruption}

Once microbes attach to a solid surface, biofilms form through microbial proliferation and the microbial production of the scaffolding extracellular matrix. Evolution of these matrices confers a survival advantage as immature biofilms are more antimicrobial susceptible than mature biofilms (43). This resistance with maturation is largely due to the deposition of EPS, which act as diffusion barriers to antimicrobial agents. Furthermore, cell-tocell communication between microbes aides in resistance development through clonal gene expression changes. Efforts have thus been put forth to disrupt biofilm matrices from maturing and preventing microcolony feedback between microbes. Methods to disrupt these matrices include enzymatic disruption, nutrient deprivation, inhibition of quorum-sensing signals and more recently mechanical disruption through the generation of air bubbles.

One method to destabilize EPS includes enzymatic disruption of the fibrin deposits that act as central structural components of the biofilm. Kwiecinski et al. (44) recently demonstrated a reduction in biofilm formation through impaired adhesion and biomass accumulation associated with the application of a tissue plasminogen activator (tPA). The investigators compared tPA-coated versus noncoated coverslips placed in the flanks of mice following a two-hour exposure of the coverslips to staphylokinasesecreting S. aureus strains. Three days after implantation, mice were euthanized and the coverslips were examined for biofilm formation. Following explantation, fewer CFUs were attached to the tPA-coated coverslips; however, the difference in bacterial attachment did not differ significantly until tPA-coating was used with antibiotic administration following implantation. Similar findings were found in vitro following a 30-minute exposure of tPA-coated polystyrene plate exposure to a bacterial suspension containing. aureus. While the utility of a tpa-coating is limited due to the presence of endogenous plasminogen activator inhibitor (PAI), which neutralizes tPA, an important proof-of-concept was established for the protective role of enzymes in the prevention of biofilm formation.

Mechanical disruption represents another method of biofilm disruption. First developed as a gas-filled micrometer-sized particle encapsulated by a stabilizing shell of either lipids, proteins, or other polymers with the intent of increasing ultrasonography resolution, microbubbles gained additional diagnostic and therapeutic indications in multiple disciplines of medicine. Microbubble-assisted ultrasonography now allows targeted drug delivery $(45,46)$, delineates anatomy intraoperatively $(47)$, facilitates gene therapy through alterations in cell membrane permeability (48), and creates the cavitations necessary for mechanical disruption of biologic fluid and tissue membrane interfaces (Figure 2) (49). Li et al. (50) recently compared ultrasound targeted microbubble destruction (UTMD) to the combined effect of UTMD + cationic antimicrobial peptide, Human $\beta$-3-defensin 3 (HBD-3), on S. epidermidis and $S$. aureus biofilm coated titanium plates implanted into a mouse model. Three days after treatment with either ultrasound (US) alone, UTMD or UTMD+HBD-3 at various doses, the number of viable colony forming units (CFU) and biofilm densities were compared. The number of viable CFUs per square centimeter significantly decreased in biofilms treated 


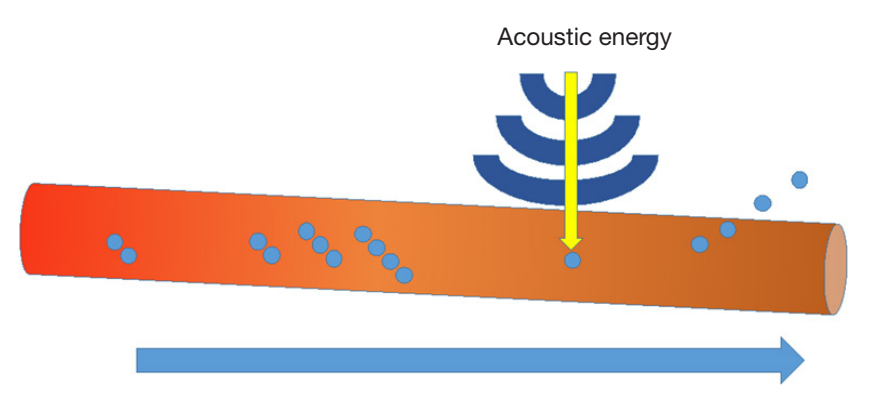

Figure 2 Intravenous microbubbles pass ultrasound waves inducing microbubble cavitation and penetration of vasculature to allow penetration into extravascular space.

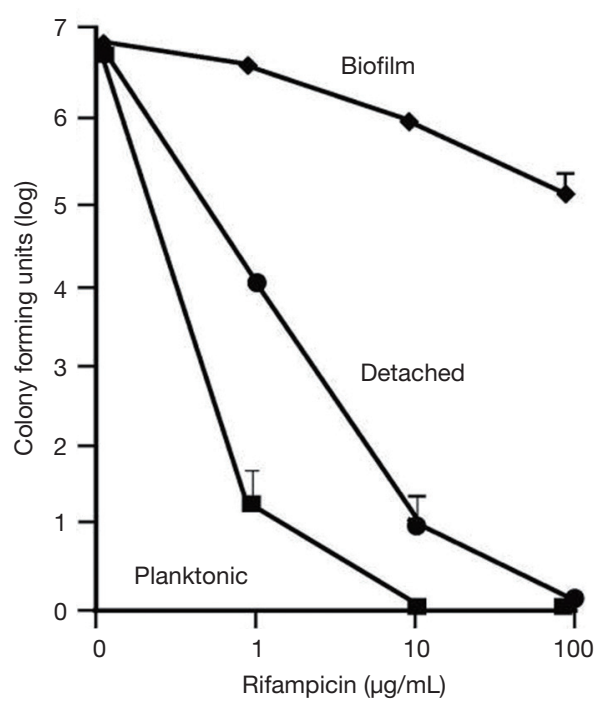

Figure 3 Susceptibility of biofilm, detached bacteria, and planktonic bacteria to Rifampicin. Adapted from Boles and Horswill (53) used under CC BY 4.0 (http://creativecommons.org/ licenses/by/4.0/).

with HBD-3 + US and HBD-3 + UTMD, with the lowest CFUs observed in the cohort of mice treated with HBD-3 and UTMD. The authors showed enhanced antimicrobial activity of HBD-3 with the addition of UTMD. However, the degree of the air bubble's mechanical insult effect depends on biofilm age and biofilm thickness and additional studies need to be performed to determine whether microbubbles and antimicrobial peptides will play a role in preventing and treating biofilms attached to IPPs.

\section{Biofilm dispersion-inducing agents}

Environmental cues and stress states (such as nutrient deprivation, excessive waste product accumulation, nitrogen and oxygen deprivation) induce biofilm dispersion and release of microbes $(43,51)$. Therefore, strategies to coax biofilm-coated microbes to shed their protective coating represent another method for biofilm control. Dispersion-based strategies have utilized genetic regulation of various intracellular signal transducers and activation of endogenous EPS enzymes integral to the dispersion of established biofilms. In an analysis of genes necessary for the dispersion of Pseudomonas putida using a transposon screen, Gjermansen et al. (52) identified the LapG protein as a member of outer membrane transglutaminases-like cysteine proteinase family that modify bacterial surface structures. This family of proteins is critical to biofilm formation as $P$. putida mutants lacking the lapD gene are not able to form biofilm. In a subsequent study by Gjermansen et al. (51), LapG and LapA protein function were modulated by altering intracellular c-diGMP levels to determine the two protein's impact on biofilm formation and eventual dispersion. The authors found increased LapG proteinase activity under lowered c-di-GMP levels resulted in increased LapA protein digestion. Moreover, loss of LapA protein, which normally functions as a surface adhesin protein and biofilm matrix component, led to dispersal of P. putida. Reduction of c-di-GMP represents and LapA protein activity therefore represents mechanisms to promote dispersion; however, this mechanism is Psuedomonas specific.

Unlike Pseudomonas, active quorum-sensing in S. aureus prevents biofilm formation (53). Quorum-sensing in $S$. aureus is controlled by the accessory gene regulator (arg) locus. The agr locus of $S$. aureus constitutes a system of transcriptional regulators that control virulence-associated genes and communication molecules that are both produced and self-sensed, called autoinducing peptides (AIP) (54). The $a g r$ system activates with glucose depletion and the introduction of autoinducing peptides (AIP). Boles and Horswill (53) demonstrated that the administration of exogenous AIP to wild-type strains of $S$. aureus resulted in biofilm sloughing using a confocal laser scanning microscope. More importantly, S. aureus that detached from their biofilm regained susceptibility to antibiotic exposure (Figure 3). Thus, disruption of $\arg$ gene function of S. aureus 
represents another avenue to induce biofilm dispersion.

\section{Conclusions}

Biofilms are three-dimensional communities of microbes that can attach to an implant surface. Biofilms confer antimicrobial resistance enhancing microbial survival in hostile environments, damage surrounding tissues, and trigger inflammation. Unfortunately, biofilms can form on IPPs and represent a feared complication of penile prosthesis surgery. Despite the progress made in aseptic technique and device coating, the infection rates remain approximately $1-3 \%$. Current management of IPP infections often necessitates device removal for successful eradication of the biofilm. However, several promising antibiofilm strategies are under development that may someday circumvent the need for device explantation. Efficient and effective methods are urgently needed beyond those available to prevent and treat biofilm formation.

\section{Acknowledgements}

The authors would like to thank Dr. Joseph Gabrielsen for his editorial assistance.

\section{Footnote}

Conflicts of Interest: The authors have no conflicts of interest to declare.

\section{References}

1. Lee DJ, Najari BB, Davison WL, et al. Trends in the Utilization of Penile Prostheses in the Treatment of Erectile Dysfunction in the United States. J Sex Med 2015;12:1638-45.

2. Hatzimouratidis K, Salonia A, Adaikan G, et al. Pharmacotherapy for erectile dysfunction: recommendations from the fourth international consultation for sexual medicine (ICSM 2015). J Sex Med 2016;13:465-88.

3. Levine LA, Becher E, Bella A, et al. Penile prosthesis surgery: current recommendations from the international consultation on sexual medicine. J Sex Med 2016;13:489-518.

4. Wilson SK, Delk JR, Salem EA, et al. Long-term survival of inflatable penile prostheses: single surgical group experience with 2,384 first-time implants spanning two decades. J Sex Med 2007;4:1074-9.
5. Wysowski DK, Swann J. Use of medications for erectile dysfunction in the United States, 1996 through 2001. J Urol 2003;169:1040-2.

6. Mehta HB, Dimou F, Adhikari D, et al. Comparison of Comorbidity Scores in Predicting Surgical Outcomes. Med Care 2016;54:180-7.

7. Beilan J, Strakosha R, Palacios DA, et al. The Postoperative Morbidity Index: a quantitative weighing of postoperative complications applied to urological procedures. BMC Urol 2014;14:1.

8. Henry GD, Wilson SK. Updates in inflatable penile prostheses. Urol Clin North Am 2007;34:535-47, vi.

9. Wilson SK, Costerton JW. Biofilm and penile prosthesis infections in the era of coated implants: a review. J Sex Med 2012;9:44-53.

10. Welliver Jr RC, Hanerhoff BL, Henry GD, et al. Significance of biofilm for the prosthetic surgeon. Curr Urol Rep 2014;15:411.

11. He W, Wang D, Ye Z, et al. Application of a nanotechnology antimicrobial spray to prevent lower urinary tract infection: a multicenter urology trial. J Transl Med 2012;10 Suppl 1:S14.

12. Rodrigues LR. Inhibition of bacterial adhesion on medical devices. Adv Exp Med Biol 2011;715:351-67.

13. Holland B, Kohler T. Minimizing Penile Implant Infection: A Literature Review of Patient and Surgical Factors. Curr Urol Rep 2015;16:81.

14. Carson CC 3rd. Efficacy of antibiotic impregnation of inflatable penile prostheses in decreasing infection in original implants. J Urol 2004;171:1611-4.

15. Eid JF. Penile Implant: Review of a "No-Touch" Technique. Sex Med Rev 2016;4:294-300.

16. Eid JF, Wilson SK, Cleves M, et al. Coated implants and "no touch" surgical technique decreases risk of infection in inflatable penile prosthesis implantation to $0.46 \%$. Urology 2012;79:1310-5.

17. Gross MS, Phillips EA, Carrasquillo RJ, et al. Multicenter Investigation of the Micro-Organisms Involved in Penile Prosthesis Infection: An Analysis of the Efficacy of the AUA and EAU Guidelines for Penile Prosthesis Prophylaxis. J Sex Med 2017;14:455-63.

18. Bjarnsholt $T$, Alhede $M$, Alhede $M$, et al. The in vivo biofilm. Trends Microbiol 2013;21:466-74.

19. Klausen M, Heydorn A, Ragas P, et al. Biofilm formation by Pseudomonas aeruginosa wild type, flagella and type IV pili mutants. Mol Microbiol 2003;48:1511-24.

20. Folsom JP, Richards L, Pitts B, et al. Physiology of Pseudomonas aeruginosa in biofilms as revealed by 
transcriptome analysis. BMC Microbiol 2010;10:294.

21. Kaplan JB, Ragunath C, Ramasubbu N, et al. Detachment of Actinobacillus actinomycetemcomitans biofilm cells by an endogenous beta-hexosaminidase activity. J Bacteriol 2003;185:4693-8.

22. López D, Vlamakis H, Kolter R. Biofilms. Cold Spring Harb Perspect Biol 2010;2:a000398.

23. Ben Jacob E, Becker I, Shapira Y, et al. Bacterial linguistic communication and social intelligence. Trends Microbiol 2004; 12:366-72.

24. Xavier KB, Bassler BL. LuxS quorum sensing: more than just a numbers game. Curr Opin Microbiol 2003;6:191-7.

25. Costerton JW, Montanaro L, Arciola CR. Biofilm in implant infections: its production and regulation. Int $\mathrm{J}$ Artif Organs 2005;28:1062-8.

26. Hoffman LR, D'Argenio DA, MacCoss MJ, et al. Aminoglycoside antibiotics induce bacterial biofilm formation. Nature 2005;436:1171-5.

27. Wilson SK, Delk JR 2nd. Inflatable penile implant infection: predisposing factors and treatment suggestions. J Urol 1995;153:659-61.

28. Arciola CR, Campoccia D, Speziale P, et al. Biofilm formation in Staphylococcus implant infections. A review of molecular mechanisms and implications for biofilmresistant materials. Biomaterials 2012;33:5967-82.

29. Campoccia D, Montanaro L, Arciola CR. A review of the biomaterials technologies for infection-resistant surfaces. Biomaterials 2013;34:8533-54.

30. Poncin-Epaillard F, Vrlinic T, Debarnot D, et al. Surface treatment of polymeric materials controlling the adhesion of biomolecules. J Funct Biomater 2012;3:528-43.

31. Ruggieri MR, Hanno PM, Levin RM. Reduction of bacterial adherence to catheter surface with heparin. J Urol 1987;138:423-6.

32. Arciola CR, Caramazza R, Pizzoferrato A. In vitro adhesion of Staphylococcus epidermidis on heparinsurface-modified intraocular lenses. J Cataract Refract Surg 1994;20:158-61.

33. Nagaoka S, Kawakami H. Inhibition of bacterial adhesion and biofilm formation by a heparinized hydrophilic polymer. ASAIO J 1995;41:M365-8.

34. Lange D, Elwood CN, Choi K, et al. Uropathogen interaction with the surface of urological stents using different surface properties. J Urol 2009;182:1194-200.

35. Arciola CR, Bustanji Y, Conti M, et al. Staphylococcus epidermidis-fibronectin binding and its inhibition by heparin. Biomaterials 2003;24:3013-9.

36. Mitik-Dineva N, Wang J, Truong VK, et al. Escherichia coli, Pseudomonas aeruginosa, and Staphylococcus aureus attachment patterns on glass surfaces with nanoscale roughness. Curr Microbiol 2009;58:268-73.

37. Kazemzadeh-Narbat M, Kindrachuk J, Duan K, et al. Antimicrobial peptides on calcium phosphate-coated titanium for the prevention of implant-associated infections. Biomaterials 2010;31:9519-26.

38. Jenssen H, Hamill P, Hancock RE. Peptide antimicrobial agents. Clin Microbiol Rev 2006;19:491-511.

39. Vecitis CD, Zodrow KR, Kang S, et al. Electronicstructure-dependent bacterial cytotoxicity of single-walled carbon nanotubes. ACS Nano 2010;4:5471-9.

40. Etcheverry-Giadrosich B, Torremade-Barreda J, PujolGalarza L, et al. Bacterial colonization of penile prosthesis after its withdrawal due to mechanical failure. Actas Urol Esp 2017;41:652-5.

41. Zeraik AE, Nitschke M. Biosurfactants as agents to reduce adhesion of pathogenic bacteria to polystyrene surfaces: effect of temperature and hydrophobicity. Curr Microbiol 2010;61:554-9.

42. Luna JM, Rufino RD, Sarubbo LA, et al. Evaluation antimicrobial and antiadhesive properties of the biosurfactant Lunasan produced by Candida sphaerica UCP 0995. Curr Microbiol 2011;62:1527-34.

43. Yang L, Liu Y, Wu H, et al. Combating biofilms. FEMS Immunol Med Microbiol 2012;65:146-57.

44. Kwiecinski J, Na M, Jarneborn A, et al. Tissue Plasminogen Activator Coating on Implant Surfaces Reduces Staphylococcus aureus Biofilm Formation. Appl Environ Microbiol 2015;82:394-401.

45. Huang C, Zhang H, Bai R. Advances in ultrasoundtargeted microbubble-mediated gene therapy for liver fibrosis. Acta Pharm Sin B 2017;7:447-52.

46. Lee S, Al-Kaabi L, Mawart A, et al. Ultrasound-mediated drug delivery by gas bubbles generated from a chemical reaction. J Drug Target 2018;26:172-81.

47. Chandra A, Gupta V, Rahul R, et al. Intraoperative ultrasonography of the biliary tract using saline as a contrast agent: a fast and accurate technique to identify complex biliary anatomy. Can J Surg 2017;60:316-22.

48. Nie F, Xu HX, Lu MD, et al. Anti-angiogenic gene therapy for hepatocellular carcinoma mediated by microbubble-enhanced ultrasound exposure: an in vivo experimental study. J Drug Target 2008;16:389-95.

49. Delalande A, Kotopoulis S, Postema M, et al. Sonoporation: mechanistic insights and ongoing challenges for gene transfer. Gene 2013;525:191-9.

50. Li S, Zhu C, Fang S, et al. Ultrasound microbubbles 
enhance human beta-defensin 3 against biofilms. J Surg Res 2015;199:458-69.

51. Gjermansen M, Nilsson M, Yang L, et al. Characterization of starvation-induced dispersion in Pseudomonas putida biofilms: genetic elements and molecular mechanisms. Mol Microbiol 2010;75:815-26.

52. Gjermansen M, Ragas P, Sternberg C, et al. Characterization of starvation-induced dispersion

Cite this article as: Herati AS, Lo EM. Penile prosthesis biofilm formation and emerging therapies against them. Transl Androl Urol 2018;7(6):960-967. doi: 10.21037/tau.2018.09.05 in Pseudomonas putida biofilms. Environ Microbiol 2005;7:894-906.

53. Boles BR, Horswill AR. Agr-mediated dispersal of Staphylococcus aureus biofilms. PLoS Pathog 2008;4:e1000052.

54. Traber KE, Lee E, Benson S, et al. agr function in clinical Staphylococcus aureus isolates. Microbiology 2008;154:2265-74. 